\title{
Zum Verhalten des Stickoxydes in höherer Temperatur
}

(II. Mittheilung)

von

F. Emich.

Aus dem chemischen Laboratorium der k. k. technischen Hochschule in Graz.

(Mit 1 Textfigur.)

(Vorgelegt in der Sitzung am 17. Juni 1892.)

Kürzlich habe ich gezeigt, wie man Stickoxyd in zugeschmolzenen Glasröhren unter Anwendung von elektrisch glühenden Metallspiralen, welche »eine dem Schmelzpunkte des Platins nahe Temperatur" besitzen, vollständig zerlegen kann. ${ }^{1}$ Bei Zusammenstellung der Arbeit wurde leider übelsehen, dass vor mir schon C. Langer und V. Meyer das erwähnte Gas durch Hitze gespalten haben; ${ }^{2}$ sie geben die dazu erforderliche Temperatur zu $1690^{\circ}$ - circa $100^{\circ}$ unter dem Schmelzpunkte des Platins - an und verwenden zur Erzeugung derselben einen eigens construirten Ofen, in welchem der (gegen $2 \mathrm{~kg}$ schwere) Platinapparat eingesetzt ist.

Wenn ich nun also selbstverständlich auf die Priorität der Ermittlung jener Zersetzungstemperatur keinen Anspruch habe, so glaube ich doch, dass meine diesbezüglichen Untersuchungen desshalb nicht ganz werthlos sind, weil sie gezeigt haben, wie man zu dem von Langer und Meyer gefundenen Resultat

1 Monatshefte für Chemie 1892, S. 78.

2 Carl Langer und Victor Meyer, pyrochemische Untersuchungen (Braunschweig 1885), S. 66. - Hern Geh. Rath Prof. V. Meyer, der mich auf mein Versehen aufmerksam machte, bin ich für seine Mittheilung zu grossem Danke verpflichtet. 
mittelst der einfachsten, in jedem Laboratorium vorhandenen Hilfsmittel gelangen kann.

Ich muss übrigens aus einem anderen Grunde auf das Verhalten des Stickoxydes in der Hitze zurückkommen.

Langer und V. Meyer behaupten a. a. O., dass das Stickoxyd beim Leiten durch Porzellanröhren, die auf $900^{\circ}$ oder $1200^{\circ} 1$ erhitzt werden, »vollständig unverändert« bleibe.

Diese Mittheilung muss desshalb überraschen, weil nach einer Abhandlung von Berthelot aus dem Jahre $1873^{2}$ Stickoxyd, das in geschlosserien Röhren auf $\mathbf{5 2 0 ^ { \circ }}$ erhitzt wird, beginnende Zersetzung zeigen soll. Wenn ich nun vorläufig von meiner Beobachtung," dass das gelbglühende Porzellanröhren verlassende Stickoxyd braune Farbe zeigt, absehe, so könnten Langer's undV. Meyer's gleichzeitig mitBerthelot's Angaben nur dann richtig sein, wenn dem strömenden Stickoxyd theilweise wesentlich andere chemische Eigenschaften zukämen als wie dem ruhenden. Dieser Fall wäre mit Rücksicht auf den Temperaturabstand von fast $700^{\circ}(1200-520)$ einzig in seiner Art und würde daher ein ganz aussergewöhnliches Interesse beanspruchen. Aus diesem Grunde erschien eine Überprüfung des Verhaltens von unserem Gase bei Temperaturen zwischen $520^{\circ}$ und $1200^{\circ}$ dringend geboten.

\section{I.}

Beim Erhitzen vom Stickoxyd in Einschmelzröhren habe ich mit Bezug auf das qualitative Verhalten jene Erscheinungen beobachtet, welche Berthelot's Angaben entsprechen. Um daher nicht Bekanntes zu wiederholen, bemerke ich nur, dass das Gas, welches sechs bis sieben Stunden im Verbrennungsofen bis zum eben beginnenden Glühen (Beobachtung im dunklen Zimmer!) erwärmt wurde, deutlich gelbliche Färbung zum Zeichen der beginnenden Zersetzung annimmt

1 Unter $900^{\circ}$, beziehungsweise $1200^{\circ}$ sind die Temperaturen zu verstehen, welche ein in dem Fletcher'schen Röhrenofen befindliches Porzellanroh: annimmt, wenn man denselben ohne, respective mit Gebläse wirken lässt. S. Langer und Meyer, 1. c. S. 48 und 51.

2 Compt. rend. 77, 1448, Chem. Centralb1. 1874, 82.

3 L. c. S. 79. 
und dann auch von Ferrosulfatlösung nicht mehr vollständig absorbirt wird.

Beispielsweise hinterliessen $28.0 \mathrm{~cm}^{3}$ Stickoxyd, welche $7^{\text {h }}$ in der angegebenen Art erhitzt wurden, $0.7 \mathrm{~cm}^{3}$ oder $3 \%$ in Eisenvitriol Unlösliches, während das angewandte Gas bis auf $0.3 \%$ vom Ferrosalz aufgenommen wurde.

II.

Langer und V. Meyer haben das Gas, welches aus dem auf $900^{\circ}$ und $1200^{\circ}$ erhitzten Rohr trat, im Absorptionsrohr aufgefangen (Sperrflüssigkeit ist nicht angegeben) und mit Pyrogallol geprüft.

Dieser Weg scheint mir nicht ganz einwandfrei. Es musste doch auch mit der Möglichkeit gerechnet werden, dass nur ein kleiner Theil des Stickoxydes in seine Bestandtheile zerfiel; dann konnte aber kein freier Sauerstoff gefunden werden, denn dieser tritt erst auf, wenn mehr als die Hälfte des Stickoxyds in $\mathrm{N}_{2}+\mathrm{O}_{2}$ gespalten wird. Ausserdem ist bei dieser Versuchsanordnung durch den Einfluss der Sperrflüssigkeit eine Täuschung möglich, denn Wasser oder Quecksilber wirken ja sehr rasch auf etwa gebildetes Salpetrigsäureanhydrid ein.

Ich habe desshalb ein Verfahren gewählt, bei dem erstens schon kleine Mengen von dissociirtem Stickoxyd sicher erkannt werden konnten und bei dem zweitens keine Sperrflüssigkeit zur Anwendung kam. Diesen Ansprüchen genügt der folgende

\section{Apparat.}

Das aus Quecksilber + Natriumnitrit + Schwefelsäure ${ }^{1}$ entwickelte, mit festem Ätzkali gereinigte Stickoxyd passirt im langsamen Strome - 20 bis 30 Blasen in der Minute entsprechend - zunächst ein $9 \mathrm{~mm}$ weites, innen glasirtes Porzellanrohr $A$, von welchem ein $30 \mathrm{~cm}$ langes Stück im Fletcher-Ofen erhitzt werden kann. Von hier gelangt es in die Beobachtungsröhre $B$, deren Enden $s^{\prime}$ und $s^{\prime \prime}$ mit Spiegelglasplatten verschlossen sind. $M N$ und $M^{\prime} N^{l}$ stellen weisse Cartonschirme vor, von denen der eine zwei kreisrunde Ausschnitte besitzt. Aus $B$ leitet man das Gas in beiderseits ausgezogene Röhren $\rho$, in denen es für die weitere Prüfung gesammelt wird.

1 Monatshefte für Chemie 1892, S. 73. 
Sieht man in der Richtung $a b$ durch die $64 \mathrm{~cm}$ lange Gasschichte, so können sehr kleine Mengen von dissociirtem Stickoxyd an der auftretenden gelben bis braunen Farbe erkannt werden. Zur Controle dient auch gelegentlich das bekannte, charakteristische Absorbtionsspectrum des Stickstofftrioxyds.

Um von dem Dissociationsgrad eine be ilä ufige Vorstellung zu gewinnen, wird die Farbe der Schichte $s^{\prime} s^{\prime \prime}$ mit der Farbe der Dämpfe verglichen, welche beim Erwärmen von Arsenik mit Salpetersäure entstehen. Dieses rohe Salpetrigsäureanhydrid

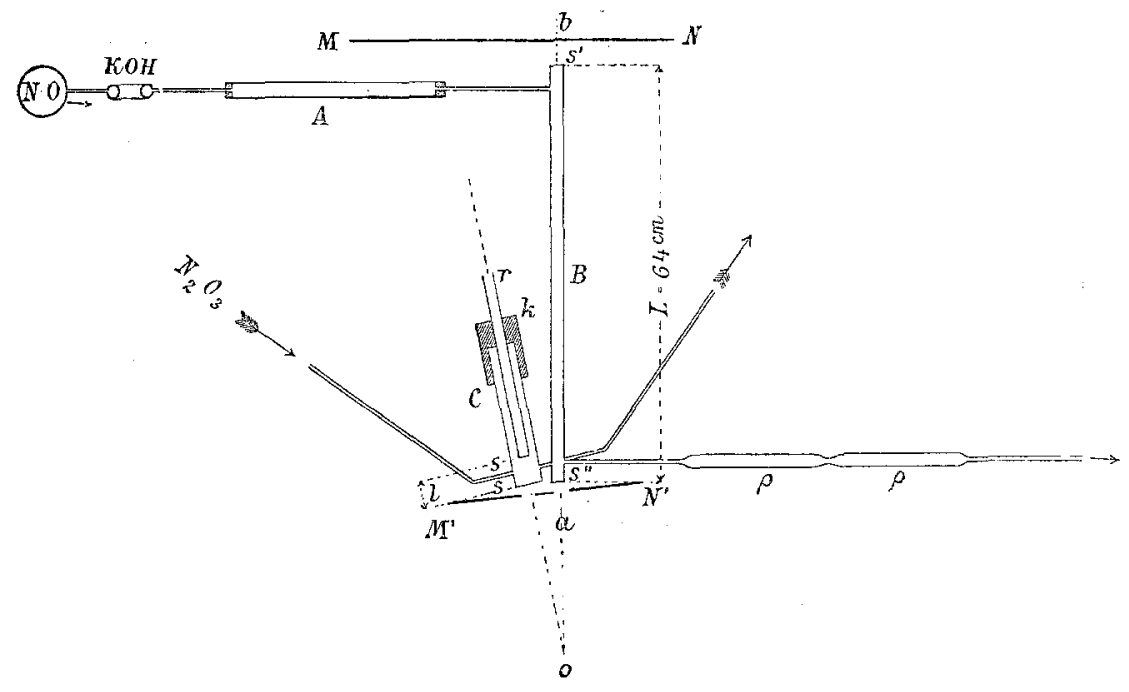

Fig. 1.

leite ich durch die Vergleichsröhre $C$ (in der man sofort eine Nachbildung von Hermann's Haemoskop erkennt). Indem man das Rohr $r$ im Korke $k$ in der Richtung seiner Längsaxe verschiebt, kann die Färbung des Trioxyds in Übereinstimmung mit der des dissociirten Stickoxyds im Rohr $B$ gebrachtwerden. Das beobachtende Auge befindet sich selbstverständlich in $O$.

Im Folgenden ist einfachheitshalber vorausgesetzt, dass der Querschnitt der Gassäulen $1 \mathrm{~cm}^{2}$ betrage.

Bedeutet $l$ die Länge der Schichte Stickstofftrioxyd, welche dieselbe Färbung zeigt, wie die Schichte $L$ des Gemisches Stickstoff + Salpetrigsäureanhydrid + Stickoxyd, entstanden 
durch Dissociation von reinem Stickoxyd, so ist unter der Annahme, dass die Zersetzung im Sinne der Gleichung

$$
\begin{aligned}
& 6 \mathrm{NO}=\mathrm{N}_{2}+2 \mathrm{~N}_{2} \mathrm{O}_{3} \\
& 6 \text { Vol. } 1 \text { Vol. } \quad 2 \text { Vol. }
\end{aligned}
$$

verläuft, $3 \mathrm{lcm}$ jene Stickoxydmenge, aus welcher $l \mathrm{~cm}^{3} \mathrm{~N}_{2} \mathrm{O}_{3}$ hervorgegangen sind.

$L \mathrm{~cm}^{3}$ dissociirtes Stickoxyd (x NO$\left.+\mathrm{N}+\mathrm{N}_{2} \mathrm{O}_{3}\right)$ entsprechen $L+\frac{3}{2} l \mathrm{~cm}^{3}$ ursprünglichem Gas. ${ }^{1}$

Es ist also das Verhältniss von der Menge des dissociirten Stickoxyds zu der des ursprünglichen — derDissociationsgrad -

$$
\varphi=\frac{3 l}{l+\frac{3}{2} l}=\frac{6 l}{2 L+3 l} .
$$

Bei dieser Ableitung sind stillschweigend zwei Voraussetzungen gemacht worden, die nicht ganz zutreffen. Einmal wurde auf die Bildung von Stickstoffoxydul, welche Berthel ot (1. c.) beim Erhitzen von Stickoxyd beobachtet hat, nicht Rücksicht genommen und weiters wurde supponirt, dass aus Arsenik-Salpetersäure reines Trioxyd entbunden werde, was bekanntlich nicht der Fall ist. Weil es sich bei den Versuchen indess nur um ganz approximative Werthe handelt, kommen die so entstehenden Fehler wohl nicht in Betracht.

\section{Versuche bei Temperaturen bis zu circa $900^{\circ}$ (Fletcher- ofen ohne Gebläse).}

Wenn der beschriebene Apparat in Gang gesetzt wird, so erkennt man in dem $64 \mathrm{~cm}$ langen Beobachtungsrohr den Beginn einer Gelbfärbung lange bevor der Ofen die ohne

1 Es ist nämlich:

Volumen von Stickstoff + Trioxyd + Stickoxyd $\ldots \ldots \ldots \ldots L \mathrm{~cm}^{3}$

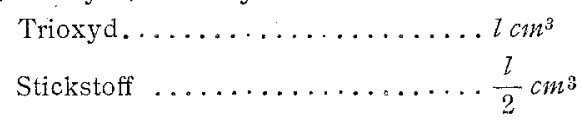

daher Volumen vom Stickoxyd, das nicht dissociirte .......L- $\frac{3}{2} l \mathrm{~cm}^{3}$ $l \mathrm{~cm}^{3}$ Trioxyd sind hervorgegangen aus Stickoxyd........ $3 \mathrm{lcm}$

daher Volumen des ursprünglichen Stickoxyds .........L- $\frac{3}{2} l+3 l \mathrm{~cm}^{3}$. 
Gebläse erzielbare Maximaltemperatur gibt; ist dieser Hitzegrad aber erreicht, so erscheintbesagte Schichte hell bra un. Beim Vergleich mit dem Salpetrigsäureanhydrid-Rohr werden in drei Versuchen folgende zufällig ganz übereinstimmende Werthe gefunden:

a) beim dunklen Rothglühen (circa $700^{\circ}$ ) $\ldots l=1 \mathrm{~mm}$

b) bei circa $900^{\circ} \ldots \ldots \ldots \ldots \ldots \ldots \ldots l=4 m$

in allen Fällen war bekanntlich $\ldots \ldots \ldots . L=640 \mathrm{~mm}$

Daraus ergibt sich das Verhältniss

$$
\begin{array}{cc}
\text { bei circa } 700^{\circ} & \text { bei circa } 900^{\circ} \\
\text { P...... } 1: 200 & 1: 50 .
\end{array}
$$

Von dem unter b) erhaltenen Gas werden Proben gesammelt, welche man auf ihr Verhalten zu concentrirter Eisenvitriollösung prüft, indem man die eine Spitze einer der Röhren $\rho$ (siehe die Figur) unterQuecksilber abbricht, das Absorbtionsmittel (circa $5 \mathrm{~cm}^{5}$ ) aufsteigen lässt, nach beendeter Absorbtion den Stand markirt und endlich die Volumina durch Auswägen ermittelt.

Gefunden wirde:

Volumen des nicht Absorbirbaren (Stickstoff) ...v=0.2 $\mathrm{cm}^{3}$ Gesammtvolumen ..............

Nimmt man die Zersetzung wie oben nach Schema $A$ ) an, so entsprechen $v$ Volumen Stickstoff $6 v$ Stickoxyd; es ist also das Verhältniss der Mengen des dissociirten zum ursprünglich vorhandenen Gesammtstickoxyd

$$
=\frac{6 v}{V+3 v} .
$$

Mit Rücksicht darauf, dass im ursprünglichen Gas $0 \cdot 4 \%$ in Eisenvitriol Unlösliches enthalten waren, findet man:

$$
\varphi=1: 35 \text {. }
$$

Die Übereinstimmung zwischen den nach ganz verschiedenen Methoden gefundenen Werthen ist wohl eine genügende für die in Betracht kommenden Umstände. 
Jedenfalls ist aus diesen Versuchen zu schliessen, dass die Zersetzung des Stickoxyds beim Leiten durch auf circa $900^{\circ}$ erhitzte Porzellanröhren noch sehr unbedeutend erscheint; sie erstreckt sich höchstens auf einige Percente und es ist recht erklärlich, dass Langer und V. Meyer dieselbe übersehen konnten.

\section{Versuche bei circa $1200^{\circ}$ (Fletcherofen mit Gebläse).}

Wird das mehrfach erwähnte Porzellanrohr mittelst Fletcherofen mit Gebläse erhitzt, so zeigt sich das austretende Stickoxyd schon in gewöhnlichen, $4-5 m m$ weiten Glasröhren deutlich gelb gefärbt. Im $64 \mathrm{~cm}$ langen Beobachtungsrohr erscheint es so dunkelbraunroth, dass eine colorimetrische Bestimmung des Dissociationsgrades wenig Aussicht bietet. Es wird desshalb der Inhalt zweier, von verschiedenen Versuchen stammenden Röhren $\rho$ mit Eisenvitriollösung wie oben geprüft. Man findet:

$$
\begin{array}{cc}
\text { Rohr I. } & \text { Rohr II. } \\
v \ldots \ldots \ldots \ldots 2 \cdot 1 \mathrm{~cm}^{3} & 4 \cdot 6 \mathrm{~cm}^{3} \\
V \ldots \ldots \ldots \ldots .4 \mathrm{~cm}^{3} & 30 \cdot 8 \mathrm{~cm}^{3}
\end{array}
$$

Daraus berechnen wir den Dissociationsgrad

$$
\begin{array}{cc}
\text { I } & \text { II } \\
1 & 0 . \ldots \ldots 0.35
\end{array}
$$

d. h. es waren im ersten Falle etwa ein Drittel, im zweiten aber an sechzig Percent des Stickoxyds im Sinne des Schemas A) zerfallen. (Beim zweiten Versuche war thatsächlich die Temperatur eine anscheinend höhere).

Wenn ich noch bemerke, dass das Stickoxyd vor dem Erhitzen des Porzellanrohrs im $64 \mathrm{~cm}$-Rohr keine Spur einer Färbung zeigte, dass man ihm aber jeden beliebigen Farbenton vom eben erkennbaren Gelb bis zum tiefen Rothbraun durch entsprechende Steigerung der Temperatur geben konnte, so wird man zugeben, dass eine Täuschung bei meinen Versuchen völlig ausgeschlossen ist. 
Es braucht kaum nochmals hervorgehoben zu werden, dass die mitgetheilten numerischen Daten für den Dissociationsgrad nur ganz relative Werthe haven. Sie wurden einzig und allein angegeben, um mit absoluter Sicherheit zu beweisen, dass die Angabe von C. Langer undV.Meyer, $\gg$ das Stickoxyd bleibt beim Erhitzen a uf $900^{\circ}$ und $1200^{\circ} \mathrm{C}$. unverändert«, auf ei nen Irrthum zurückzuführen ist. 